\title{
SUL «FANTASTICO» ITALIANO: TRA LA LESSICOGRAFIA E LA STORIA DI LETTERATURA
}

DOI: http://dx.doi.org/10.12775/TSP-W.2020.015

Date of receipt: 23.06 .2020

Date of acceptance: 13.11 .2020

On the Italian Fantastic: Between Lexicography and Literary History. The fantastic in literature has been discussed so thoroughly that any new paper on the subject may arouse suspicion of reworking the existing ideas. However, the aim of the present article is to fulfill a gap in the research into the fantastic literature, especially the Italian fantastic literature, and address a problem which has not been answered appropriately in this field of study. The previous research into the fantastic has almost always omitted the etymology, focusing only on the 19th-century literary origins of the term, whereas the present paper analyzes modern dictionary definitions of the term, traces the historical development of its meaning since the earliest recorded occurrence of the word in the Italian language, and finally examines minutely the Greek etymology of the word and collates it with the most recent meaning that the word acquired in the 19th century, namely fantastic as a literary term. The meticulous etymological analysis has allowed to shed light on surprising denotations and connotations of the word fantastic, which, although omitted in Italian dictionaries, remain to this day implicit in the role and the characteristics of this literature in Italy. The article is a contribution to the author's research into the contemporary Italian fantastic novel. The arguments presented in this paper may confirm the

${ }^{1}$ Mgr Patrycja Przełucka - Doctoral School of the University of Silesia,ORCID: 0000-00034157-2868, e-mail: patrycja.przelucka@us.edu.pl. 
author's thesis that the fantastic literature, and above all the Italian one, tends to serve as a tool for commenting and interpreting the reality.

Keywords: fantastic, Italian literature, etymology, lexicography.

O włoskiej ,fantastyczności”: między leksykografią a historią literatury. Fantastyczność w literaturze została omówiona tak gruntownie, że każda nowa praca na ten temat może budzić podejrzenie, że przetwarza jedynie wcześniejsze poglądy. Celem niniejszego artykułu jest jednak wypełnienie luki w badaniach nad literaturą fantastyczną, zwłaszcza włoską, i podjęcie problemu, któremu nie poświęcono do tej pory należytej uwagi. Mianowicie, w dotychczasowych badaniach nad fantastycznością w literaturze prawie zawsze pomijano etymologię słowa „fantastyczny”, koncentrując się jedynie na XIX-wiecznej literackiej genezie tego terminu. Niniejsza praca zawiera natomiast analizę jego współczesnych definicji słownikowych, ukazuje historyczny rozwój jego znaczeń od najwcześniejszego odnotowanego wystąpienia tego słowa w języku włoskim, a na koniec analizuje dokładnie grecką etymologię, zestawiając ją z najnowszym znaczeniem, jakie omawiany termin uzyskał w XIX wieku, czyli „fantastyczny” jako termin literacki. Skrupulatna analiza etymologiczna pozwala rzucić światło na zaskakujące denotacje i konotacje słowa ,fantastyczny”, które, choć pomijane w słownikach włoskich, pozostają do dziś ukryte w roli i cechach charakterystycznych tej literatury we Włoszech. Artykuł stanowi wkład w badania autorki nad współczesną włoską powieścią fantastyczną. Przedstawione $\mathrm{w}$ artykule argumenty potwierdzają tezę autorki, że literatura fantastyczna, w szczególności włoska, służy często jako narzędzie komentowania i interpretowania rzeczywistości.

Słowa kluczowe: fantastyczność, literatura włoska, etymologia, leksykografia.

La riflessione sul fantastico nella letteratura è nata nella cultura europea durante il Romanticismo. La nascita del termine, il processo della formazione delle varie accezioni del suo significato e gli inizi del dibattito attorno al tema del fantastico prendono forma proprio in quell'epoca.

Nel 1814 Ernst Theodor Amadeus Hoffmann con i suoi Fantasiestücke in Callots Manier diede vita ad una nuova estetica letteraria che dopo esser tradotto in francese nel 1829, conobbe una vera moda in Francia e riscosse un successo spettacolare. Questo nuovo tipo di letteratura fu definito come fantastic (it. 'fantastico') da Walter Scott nel suo saggio On the Supernatural in Fictitious Composition, and particularly on the Works of Ernest Theodore William Hoffmann nel $1827 .^{2}$

${ }^{2} \mathrm{Si}$ veda: SCOTT W., On the Supernatural in Fictitious Composition, and particularly on the Works of Ernest Theodore William Hoffmann, in "Foreign Quarterly Review", I, 1827, pp. 60-98. 
Il saggio, che aspramente criticò la produzione dell'autore tedesco, è stato determinante nel mettere in atto una discussione complessa su come definire questo nuovo fenomeno letterario. I francesi hanno sostanzialmente ignorato la critica morale ed estetica fornita da Scott, ma hanno adottato la sua etichetta fantastic con enorme entusiasmo ${ }^{3}$ per descrivere lo stile di Hoffmann, che, sebbene contenesse elementi soprannaturali, non era più il meraviglioso che i francesi conoscevano, ma appunto il fantastico. Fu Scott a dare inizio al dibattito critico sul fantastico coniando il termine e riconoscendo la specificità rivoluzionaria dei racconti di Hoffmann la cui interpretazione dell'inconsueto e del soprannaturale era del tutto diversa rispetto alla tradizione letteraria precedente, quella del meraviglioso. ${ }^{4}$

A cominciare da quel momento il fantastico non cessa di imporsi all'attenzione della critica letteraria, soprattutto in Italia dove il termine letterario fantastico non è ancora stato codificato a differenza della Francia o dell'Inghilterra. Infatti, per molti aspetti, il fantastico italiano sembra eludere i punti essenziali delle teorie internazionali del fantastico in letteratura, soprattutto della dominante scuola francofona. La situazione è resa ancora più complicata dal fatto che a causa della copiosità e delle discrepanze notevoli degli studi europei sull'argomento, non c'è la corrispondenza di significato tra gli equivalenti della parola fantastico nelle lingue occidentali.

L'argomento del fantastico nella letteratura è stato discusso in modo approfondito sia dagli scrittori stessi che dai critici letterari in quasi tutti i paesi occidentali. Per questo motivo, qualsiasi nuovo studio sul tema può suscitare il sospetto di rielaborare le idee già esistenti. Però, il semplice fatto che dai primi commenti su Hoffmann fino ad oggi siano state scritte tante opere contrassegnate con il marchio del fantastico così diverse tra di loro rende legittima la necessità di una definizione dettagliata e minuziosa fornita con somma diligenza e scrupolosità. Pertanto, lo scopo di quest'articolo è di affrontare una questione a cui non è stata data una risposta adeguata nelle ricerche precedenti in questo campo di studio, cioè l'analisi profonda degli inizi del fantastico. Però si intendono qui non gli inizi letterari, poiché quelli sono già stati descritti in modo dettagliato nella letteratura critica, ma le origini linguistiche ed il principio cognitivo da cui deriva il lemma fantastico. Pertanto, per colmare questo gap di ricerca, prenderò

${ }^{3}$ SAGE V., Scott, Hoffmann, and the Persistence of the Gothic, in Popular Revenants: The German Gothic and Its International Reception, 1800-2000, Andrew Cusack, Barry Murnane (a cura di), Camden House, New York, 2012, p. 84.

${ }^{4}$ LAZZARIN S., Agli albori della teoria del fantastico, in Il fantastico italiano. Bilancio critico e bibliografia commentata (dal 1980 a oggi), LAZZARIN S. (a cura di), Mondadori, Milano, 2016, p. 1. 
libertà di occuparmi al primo posto della definizione lessicografica fornita dai dizionari moderni, poi traccerò le trasformazioni storiche del significato di fantastico nella lingua italiana fin dalle origini dell'italiano come lingua nazionale.

Successivamente, intraprenderò il compito di fare approfondimenti etimologici per scoprire la parola originaria della catena etimologica che sottende a fantastico al fine di analizzare dei rapporti che la moderna accezione del termine fantastico ha con la parola originaria da cui è derivato. In fine, passerò al valore più moderno assunto da fantastico che segnò la sua apparizione come neologismo semantico nell'anno 1830 e che venne registrato dalla lessicografia negli anni 1860: cioè fantastico inteso come termine letterario.

È importante sottolineare qui che già agli albori della teoria del fantastico, quando il termine iniziò a designare una categoria letteraria, vi è apparsa una dissonanza, tuttora in voga, e la scissione del pensiero teorico in tre linee. La prima linea è quella riferita agli studiosi, come la maggioranza della scuola francofona, che scorgono nel fantastico un genere sotto forma di un breve racconto radicato storicamente negli anni Dieci e Venti dell'Ottocento ed iniziato con la produzione di E.T.A. Hoffmann. Poi c'è la linea di quelli che, come Walter Scott, vedono lo stesso fenomeno ottocentesco come un modo letterario, e finalmente c'è la linea dei teorici, come alcuni dei primi esponenti e commentatori dell'estetica come Charles Nodier e Guy de Maupassant ed anche alcuni studiosi eminenti italiani come Italo Calvino, Sergio Solmi e Gianfranco de Turris, che, considerando il fantastico un modo letterario, lo intendono come una categoria sovrastorica di tutti i generi e sottogeneri che usano lo schermo non mimetico per rivelare gli aspetti meno trasparenti della realtà. Quella è una delle tante divergenze che permeano il dibattito sul fantastico. ${ }^{5}$

Affrontando l'impegno di fornire la definizione completa di un termine e puntando sull'analisi minuziosa e dettagliata della sua evoluzione diacronica, occorre rivolgersi ad un riferimento di primaria importanza in tali casi, cioè al dizionario. Per presentare il significato moderno del lemma fantastico, un ag-

${ }^{5}$ Il presente articolo è un contributo alla ricerca più ampia sul fantastico nella letteratura italiana, in cui l'autrice mira allo scopo di dimostrare che la letteratura nazionale italiana si basa sul fantastico fin dalle origini e che il fantastico del Novecento italiano non è una forma nuova come vorrebbero alcuni studiosi francofoni e i loro seguaci, ma una fase di fioritura dell'evoluzione di quel modo letterario sovrastorico. La letteratura italiana vanta una tradizione grandiosa del fantastico che si può tracciare da Commedia di Dante, Decameron di Boccaccio e Orlando Furioso di Ludovico Ariosto alle opere di Tommaso Landolfi, Italo Calvino, Michele Mari, Antonio Tabucchi, Antonio Scurati e Laura Pugno per citarne solo alcuni. Una simile visione del fantastico nella letteratura italiana è rappresentata da Italo Calvino stesso ed è stata anche indicata da Charles Nodier già nel 1830, agli albori del dibattito europeo sul fantastico. 
gettivo che può anche essere sostantivato, utilizzerò le definizioni fornite dalle lessicografie autorevoli di Treccani, Zingarelli e Garzanti. Tra le accezioni che emergono dalle definizioni e dagli esempi forniti da questi vocabolari, il termine ne ha quattro principali che possono essere sintetizzate con punti seguenti:

1. Della fantasia; che riguarda la fantasia.

2. a) Creato dalla fantasia; concepito dalla fantasia; che è frutto di fantasia; in cui ha parte prevalente la fantasia.

b) Che non ha fondamento se non nella fantasia, quindi irreale, immaginario, chimerico; anche di oggetti o fatti reali, ma che per essere straordinarî, inconsueti, fuori della norma, sembrino creati dalla fantasia; che è prodotto dalla fantasia e non ha necessaria rispondenza nella realtà dei fatti.

3. (per iperbole, con valore superlativo) che suscita grande ammirazione; straordinario; eccezionale; favoloso.

4. (di persona) bizzarro, originale, stravagante, cervellotico, lunatico.

La definizione di Treccani comprende anche un significato addizionale secondo cui il sostantivo fantastico può denotare in araldica una figura chimerica mostruosa, composta dall' accostamento di parti del corpo di vari animali, il che sembra molto interessante, dato che la letteratura fantastica assume spesso un carattere ibrido, contaminando modi, generi ed elementi di varia provenienza.

Ora vale la pena tracciare la storia del termine fantastico nella lingua del Bel Paese, seguendo l'asse del percorso storico indietro nel tempo, scoprendo via via gli inizi della parola. Per questo scopo si è rilevato cruciale ricorrere al Dizionario della Lingua Italiana di Nicolò Tommaseo e Bernardo Bellini, il più importante dizionario storico della lingua italiana prodotto durante il Risorgimento italiano che fornisce un lemmario dell'uso per i decenni del 1860 e del 1870. Vi è compreso tutto il patrimonio della tradizione linguistica italiana dell'epoca, arricchito di citazioni di autori sette- ed ottocenteschi. Per la parola fantastico il Dizionario di Tommaseo fornisce le accezioni seguenti:

1. Della fantasia, o Dei fantasmi della mente; L'intelletto nostro non può intendere cosa alcuna, la quale non sia nella virtù (potenza) fantastica ovvero immaginativa; Associazioni fantastiche d'idee, dove un fantasma richiama l'altro, o un fantasma un'idea astratta, o l'idea il fantasma. Può la facoltà imaginativa essere più $\mathrm{o}$ meno fantastica, in senso non buono, e anche buono, cioè rappresentare fantasmi o fantasie più o meno in copia, e meno o più vivamente.

2. Nelle opere d'arte. Imitazione fantastica, che è imitatrice delle cose che non sono. $\mathrm{O}$ delle cose che sono, ma congegnate in modi diversi dal reale, o piutto- 
sto dal materiale degli oggetti esteriori. Poema, Dramma, Romanzo fantastico. Opera fantastica. Genere fantastico d'arte.

3. D’oggetti anco reali, ma che, per la singolarità loro, pajono fatture di fantasia; che in modo non sempre piacevole dánno nella fantasia di chi li vede o li pensa.

4. Quindi contrario al reale.

5. Di persona strana per movimenti di fantasia soprabbondante.

6. Degli atti della mente, e quindi della volontà. ${ }^{6}$

Come vediamo, la definizione ottocentesca di fantastico segna un significato in più rispetto alle definizioni moderne, cioè aggiunge l'accezione che la parola ha ottenuto nell'ambito letterario e ne fornisce una descrizione abbastanza ampia, spiegando che fantastico in letteratura può denotare la rappresentazione delle cose che non sono reali, ma anche la rappresentazione delle cose esistenti, rielaborate tramite la modalità di straniamento. Inoltre, indica che fantastico è un genere letterario e può essere sia un poema, un dramma che un romanzo.

Retrocedendo ulteriormente nel percorso evolutivo del campo semantico di fantastico, occorre spostarsi nel Seicento, alla lessicografia più autorevole di quel tempo e, in particolare, il primo vocabolario della lingua italiana, il Vocabolario della Crusca nel 1612. A quel tempo, nella prima metà del Seicento e probabilmente anche nel Cinquecento, il termine sembra esser appartenuto solo al campo semantico di finzione, come si può vedere nella definizione, succinta e laconica, fornita dal Vocabolario che esplica fantastico con le parole seguenti: «Da FANTASMA, finto, immaginato, non vero». ${ }^{7}$ Allora fantastico significava falso e bugiardo. Quello che più sorprende è la sfumatura assolutamente negativa della definizione ed anche la brevità della voce rispetto alle definizioni posteriori.

Un contributo di rilievo nella ricerca sulla dimensione storica del termine è fornita dal Tesoro della lingua Italiana delle Origini, un grande database testuale che raccoglie circa venti milioni di parole provenienti dai testi scritti in lingua italiana prima del 1375 sia in prosa che in poesia. Dalle sue fonti risulta che già prima del 1375, quindi prima del consolidarsi dell'italiano nazionale, il termine fantastico possedeva significati molteplici, simili a quelli odierni, ed una vasta area di distribuzione geolinguistica, apparendo nei dialetti settentrionali, centrali e meridionali. Il dizionario Zingarelli localizza il primo uso della parola prima

${ }^{6}$ TOMMASEO N., BELLINI B., Fantastico, voce in Dizionario della Lingua Italiana Nuovamente Compilato, Dalla Società L'Unione Tipografico-Editrice, Torino, 1861, Vol. II, p. 649 , http://www.bdcrusca.it/im_tom.asp?radice=000057481_3\&file_seq=658 [ultimo accesso: 29.03.2020].

${ }^{7}$ Fantastico, voce in Vocabolario della Crusca, Accademia della Crusca, Firenze, 1612, http://vocabolario. signum.sns.it [ultimo accesso: 29.03.2020]. 
del 1292, ma tralascia purtroppo di fornire la fonte, mentre secondo il Tesoro la prima attribuzione del termine risale alla Fiorita, un'ampia compilazione enciclopedica di carattere storico-leggendario, scritta da Armannino Giudice da Bologna nel 1325 dove la parola fantastico appare nella frase seguente: «Questa Sibilla che mostrò lo inferno ad Enea fu femina fantastica e avea indosso lo spirito maligno, la quale si chiama Sibilla Cumea per lo luogo dov'ella abitava». ${ }^{8} \mathrm{La}$ frase apre una descrizione del viaggio di Enea nel mondo ultraterreno. Sembra di estremo interesse notare a questo punto che sebbene la Francia con Nodier, Maupassant, Castex, Vax, Caillois e Todorov fosse famosa per le sue teorie del fantastico letterario, il lemma fantastico apparve in francese molto più tardi che in italiano, solo nel Cinquecento, ${ }^{9}$ allora due secoli dopo la composizione della Fiorita. Comunque, tornando al merito, secondo le definizioni fornite dal Tesoro, fantastico alle origini della lingua italiana significava:

1. Privo degli attributi della realtà, incorporeo, immateriale (bolognese, pisa, fiorentino, messino) 1.1 Dotato di poter soprannaturali (toscano) (questo è il significato presentato nella Fiorita)

2. Relativo a quella parte del cervello che rielabora la memoria con l'immaginazione (fiorentino) 2.1 Ottenuto con la fantasia (toscano, veneto) 2.2 Locuzione nominale del Virtù fantastica come la fantasia, l'immaginazione (Boccaccio fiorentino, pisano)

3. Frutto dell'immaginazione (in opposizione alla ragione); frutto dell'inganno e della falsità, fallace (toscano occidentale, pisano, fiorentino, toscano, senese, siciliano, fiorentino) 3.1 Privo di senno, stravagante o matto (perugiano, fiorentino, siciliano) 3.1.1. Eretico (di Lazio). ${ }^{10}$

I dizionari continuarono quindi a riprodurre la sfumatura negativa di fantastico concepito in quanto falso, finto dagli inizi della lingua italiana nazionale almeno fino al Seicento (si veda la definizione fornita dall'Accademia della Crusca).

Può sembrare strano che la maggioranza della lessicografia italiana non recepisca l'accezione letteraria del termine fantastico, ma l'abbondanza di apporti

${ }^{8}$ MAZZATINTI G., La Fiorita di Armannino Giudice, in "Giornale di filologia romanza", III, 1880, p.30.

9 LAZZARIN S., Les Mille et Une Morts du Fantastique, in "Caietele Echinox Journal", Université Jean Monnet, Saint-Étienne, no 16, 2009, p. 124.

${ }^{10}$ Fantastico, voce [in:] Tesoro della Lingua Italiana delle Origini. Il primo dizionario storico dell'italiano antico che nasce direttamente in rete, fondato da Pietro G. Beltrami, 1997, http:// tlio.ovi.cnr.it/TLIO/ [ultimo accesso: 19.02.2021]. 
teorici contraddittori sull'argomento ha reso il termine sfuggente, evanescente, perfino controverso. Allora è naturale che i dizionari tralascino questo significato perché non è facilmente definibile in italiano e occorre un commento più lungo di una voce del dizionario. Solamente il Dizionario di Tommaseo contempla il fantastico come termine letterario, ma l'elaborazione di quella definizione coincise con il primo uso del fantastico per riferirsi a specifiche opere letterarie (si veda il saggio di Scott) perciò non sorprende l'inclusione di quell'accezione nel dizionario dell'Ottocento.

Tutte le voci, sottovoci e accezioni sopraccennate dimostrano che fantastico ha assunto, con il passar del tempo, un maggiore spessore semantico. Però, il contributo più significativo nello studio del percorso storico compiuto da fantastico sarà fornito dall'etimologia che presenterà la risposta alla domanda dove la parola studiata affonda le sue radici più antiche. Tipicamente i dizionari tendono a indicare come la prima etimologia del fantastico la parola phantăsticu $(m)$ proveniente dal tardo latino, la quale significa «immaginario, irreale». Ciò giustifica la sfumatura negativa spesso presente nei significati della parola italiana. Però le lessicografie più avanzate, come quelle menzionate sopra, esplicano anche che la parola latina deriva dall'aggettivo greco phantastikós con il significato molto simile: «immaginario, inventato, fittizio, finto». La parola phantastikós, a sua volta, deriva dal verbo greco phantazo che presenta un significato del tutto imprevisto: «far vedere, mostrare».

Il solo fatto che quest'originaria accezione greca non rientri nelle denotazioni registrate nei dizionari italiani basta per indicare che ci deve essere dell'altro in fantastico, un qualcosa che costituisce i fondamenti più profondi del campo associativo del termine, ma non si manifesta in superficie. È bene rimarcare qui che la ricerca a cui quest'articolo fornisce un contributo si concentra sull'ipotesi dell'autrice che l'impegno sociale e culturale sia un elemento immanente della letteratura fantastica italiana a cui gli autori che la praticano affidino una missione di ricercare la verità, esercitare un influsso sulla realtà e comunicare un messaggio morale all'umanità. Una ricerca etimologica più approfondita del greco phantazo lascia esplorare quest'accezione apparentemente estranea allo scopo di trovare la parola originaria che aveva iniziato la catena etimologica, dando vita a fantastico italiano secoli dopo. Per conseguire questo scopo mi sono avvalsa del A Greek-English Lexicon di Henry George Liddell e Robert Scott e delle risorse del Blue Letter Bible che offrono lo studio approfondito delle parole bibliche greche in base a The New Strong's Exhaustive Concordance of the Bible, Expository Dictionary of New Testament Words di William Edwy Vine, Greek Lexicon di Joseph H. Thayer e Analytical Greek Lexicon di Wesley J. Perschbacher. 
Innanzitutto, la definizione allargata del verbo phantazo menzionato dai dizionari offre il significato seguente: «far apparire, rendere visible, rendere apparente, esporre, rivelare, mostrare». ${ }^{11}$ Però, la parola vanta anche alcune accezioni secondarie altrettanto interessanti: «rendere presente all'occhio o alla mente; mettersi qualcosa davanti alla propria mente; pitturare un oggetto a se stessi, immaginare; diventare visibile, apparire, essere ascoltato, essere terrorizzato da visioni o fantasmi; fare uno spettacolo; rendersi come qualcuno; prendere la forma di qualcuno; apparire così e così per essere immaginato; essere informato contro». ${ }^{12}$ Ulteriori indagini hanno portato alla scoperta che phantazo deriva dal verbo phaino che «significa portare alla luce, far brillare, gettare luce, dare alla luce o brillare, essere chiaro o risplendente, diventare manifesto e evidente, essere portato alla luce, apparire, essere esposto, dimostrarsi, incontrare gli occhi, colpire la vista, diventare chiari o manifestare essere visto, apparire alla mente, sembrare al proprio giudizio o opinione». ${ }^{13}$ Phaino a sua volta deriva dalla parola greca phao che sembra la parola originaria e base della catena etimologica della parola fantastico e significa «brillare, dare luce o far manifestare tramite dei raggi» ${ }^{14}$. La parola contiene le radici pha- e phan- che alludono al significato de «la luce come vista dall'occhio» e figurativamente a «la luce che raggiunge la mente». È interessante notare che dalla stessa parola-radice derivino anche il nome phos e il verbo phemi. Phos significa letteralmente «la luce o qualunque cosa che emette la luce» e figurativamente significa «la luce della verità e della sapienza; la mente e la ragione; la capacità di comprendere le verità morali e spirituali». ${ }^{15}$ Phemi significa «dire spiegando, dire esplicando, affermare, comunicare, dichiare le proprie parole». ${ }^{16}$

Come vediamo, le definizioni tratte da dizionari italiani, sia moderni che storici, non si avvicinano nemmeno lontanamente agli originari significati greci del lemma. Lo slittamento di significato può sembrare colossale, ma infatti quei

${ }^{11}$ phantazo, voce in Blue Letter Bible, Sowing Circle, https://www.blueletterbible.org/lang/ lexicon/lexicon.cfm?Strongs=G5324\&t=KJV [ultimo accesso: 19.02.2021].

${ }^{12}$ LIDDELL H.G., SCOTT R., $\varphi \alpha v \tau \alpha ́ \zeta \omega$, voce in A Greek-English Lexicon, revisionato e ampliato da Sir Henry Stuart Jones, con l'assistenza di Roderick McKenzie, Clarendon Press, Oxford, 1940.

${ }^{13}$ phaino, voce in Blue Letter Bible, Sowing Circle, https://www.blueletterbible.org/lang/ lexicon/lexicon.cfm?Strongs=G5316 [ultimo accesso: 19.02.2021].

${ }^{14}$ phōs, voce in Blue Letter Bible, Sowing Circle, https://www.blueletterbible.org/lang/lexicon/lexicon.cfm?strongs=G5457\&t=KJV [ultimo accesso: 19.02.2021].

${ }^{15} \mathrm{phos}$, voce in Blue Letter Bible, Sowing Circle, https://www.blueletterbible.org/lang/lexicon/lexicon.cfm?strongs=G5457\&t=KJV [ultimo accesso: 19.02 .2021$]$.

${ }^{16}$ phèmi, voce in Blue Letter Bible, Sowing Circle, https://www.blueletterbible.org/lang/lexicon/lexicon.cfm?strongs=G5346\&t=KJV [ultimo accesso: 19.02.2021]. 
significati originari stranamente convergano con gli scopi a cui hanno mirato gli autori italiani della letteratura nata in chiave fantastica. In questa ricerca si ritiene sostenibile una tesi che, anche se i dizionari della lingua italiana continuano a tralasciare queste accezioni primarie, la letteratura italiana del modo fantastico sorprendentemente ha continuato ad incorporarle dai suoi principi fino ad oggi, il che è visibile nell'impegno morale e sociale di questa letteratura. Quest' articolo mira a sistematizzare l'evoluzione diacronica del termine fantastico e le sue origini etimologiche, ed ha un carattere introduttivo per ulteriori studi in merito che consistono nello studiare come la letteratura fantastica in Italia, a differenza per esempio dalla produzione fantastica francese, è stata sempre una letteratura impegnata che collocandosi apparentemente fuori dalla realtà parla proprio della realtà, nel modo più efficace del mimetismo, ormai esaurito. Si vuole mostrare nelle indagini ulteriori che, proprio come indicano le definizioni greche, il fantastico nella letteratura italiana serve a rendere visibile la natura vera della realtà ed esporre i suoi fenomeni e problemi latenti.

\section{BIBLIOGRAFIA:}

Beltrami P. G. (fondato da) (1997), Tesoro della Lingua Italiana delle Origini. Il primo dizionario storico dell' italiano antico che nasce direttamente in rete, http://tlio. ovi.cnr.it/TLIO/.

Blue Letter Bible, Sowing Circle, https://www.blueletterbible.org/.

Lazzarin S. (2009), Les Mille et Une Morts du Fantastique, in "Caietele Echinox Journal", Université Jean Monnet, Saint-Étienne, no 16, pp. 124-135.

Lazzarin S. (2016), Agli albori della teoria del fantastico, in Il fantastico italiano. Bilancio critico e bibliografia commentata (dal 1980 a oggi), Lazzarin S. (a cura di), Mondadori, Milano,

Liddell H.G., Scott R. (1940), A Greek-English Lexicon, revisionato e ampliato da Sir Henry Stuart Jones, con l'assistenza di Roderick McKenzie, Clarendon Press, Oxford.

Mazzatinti G. (1880), La «Fiorita» di Armannino Giudice, in "Giornale di filologia romanza", III, 1880, pp. 1-55.

Sage V. (2012), Scott, Hoffmann, and the Persistence of the Gothic, in Popular Revenants: The German Gothic and Its International Reception, 1800-2000, Andrew Cusack, Barry Murnane (a cura di), Camden House, New York.

Scott W. (1827), On the Supernatural in Fictitious Composition, and particularly on the Works of Ernest Theodore William Hoffmann, in "Foreign Quarterly Review", I, pp. 60-98. 
Tommaseo N., Bellini B. (1861), Dizionario della Lingua Italiana Nuovamente Compilato, Dalla Società L’Unione Tipografico-Editrice, Torino, Vol. II, http://www. bdcrusca.it/im_tom.asp?radice=000057481_3\&file_seq=658.

Vocabolario della Crusca (1612), Accademia della Crusca, Firenze, http://vocabolario. signum.sns.it. 
